\title{
Low-Complexity Frequency Offset and Phase Noise Estimation for Burst-Mode Digital Transmission
}

\author{
Jabran Bhatti, Nele Noels and Marc Moeneclaey \\ Department of Telecommunications and Information Processing \\ Ghent University, Belgium \\ Email: $\{$ Jabran.Bhatti, Nele.Noels, mm $\} @$ telin.ugent.be
}

\begin{abstract}
The presence of a frequency offset (FO) and phase noise can cause severe performance degradation in digital communication systems. This work combines a simple FO estimation technique with a low-complexity phase noise estimation method, inspired by the space-alternating generalized expectation-maximization algorithm. Using a truncated discretecosine transform (DCT) expansion, the phase noise estimate is derived from the estimated DCT coefficients of the phase. A number of implementations of the proposed algorithm are discussed. Numerical results indicate that when estimating the FO from pilot symbols only, comparable performance can be reached as the computationally more complex case where the FO is updated iteratively, with small convergence time. The phase noise estimation step is well capable of compensating for the residual FO. For the considered scenario, performing FO compensation before iterative phase noise estimation yields a biterror rate performance degradation close to the case where the FO is known.
\end{abstract}

\section{INTRODUCTION AND MOTIVATION}

In an ideal bandpass digital communication system, the local oscillators required for up-conversion at the transmitter and down-conversion at the receiver operate at exactly the same nominal carrier frequency. In practice, however, there will be a mismatch between the carrier frequencies of the received signal and the sinusoid generated by the receiver oscillator. This mismatch is called the carrier frequency offset (FO). Nonideal effects in the transmitter and receiver oscillators also cause the carrier phase of the received baseband signal to show random fluctuations in time; this impairment is commonly known as phase noise and has been characterized in e.g. [1]. As a result of frequency offsets and randomly perturbed phase samples, the received signal contains a rotated version of the transmitted symbol, yielding an increased error probability. An efficient FO and phase noise estimation technique is of utmost importance to guarantee reliable transmission.

A variety of FO estimation techniques have been presented in [2]-[5]. Here, we use the space-alternating generalized expectation-maximization (SAGE) algorithm [6] to combine FO estimation based on [5] with the phase noise estimation technique from our prior work [7], which makes use of a truncated basis expansion of the phase. In section II, we describe the system and discuss the basis expansion model for the phase. Section III presents the proposed FO and phase noise estimation algorithm. Performance of the proposed technique is assessed via computer simulations in section IV, where both the mean-square phase error (MSPE) and the associated bit-error rate (BER) are discussed.

\section{SYSTEM MODEL}

We consider a single-carrier transmission system, where bursts of $K$ uncoded PSK symbols are transmitted over an additive white Gaussian noise (AWGN) channel suffering from phase noise. In the presence of a FO (under the assumption that perfect timing synchronization takes place), the matched filter output samples are given by

$$
\begin{aligned}
r_{k} & =a_{k} e^{j\left(2 \pi \nu k+\theta_{k}\right)}+w_{k} \\
& =a_{k} e^{j \phi_{k}}+w_{k},
\end{aligned}
$$

for $k=0, \ldots, K-1$. Here, $K$ is the burst length and $k$ is the symbol index. The additive noise $\left\{w_{k}\right\}$ is a sequence of i.i.d. zero-mean circular symmetric complex-valued Gaussian random variables with $E\left[\left|w_{k}\right|^{2}\right]=N_{0}$, and $\theta_{k}$ is the sum of a static phase offset $\theta_{\text {stat }}$ and a zero-mean phase noise process. $\nu$ represents the frequency offset between the transmitter and receiver oscillators, normalized to the symbol interval $T$. The FO introduces a term to the time-varying phase that increases linearly with $k$. Combining both the FO and phase noise contributions, the total instantaneous phase is modeled as $\phi_{k}=2 \pi \nu k+\theta_{k}$.

The transmitted symbol sequence $\left\{a_{k}\right\}$ contains $K_{P}$ pilot symbols at positions $k \in I_{P}=\left\{k_{i}, i=0, \ldots, K_{P}-1\right\}$, with constant magnitude: $\left|a_{k_{i}}\right|^{2}=E_{s}$. We denote the pilot symbol ratio as $\eta=K_{P} / K$. The remaining $K-K_{P}$ data symbols are chosen from a PSK constellation, with $E\left[\left|a_{k}\right|^{2}\right]=E_{s}$ for $k \notin I_{P}$. As in [7], the low-pass character of the phase noise process is exploited by representing $\theta_{k}$ by a truncated discretecosine transform (DCT) expansion, containing only $N<<K$ lower-order DCT coefficients. Due to their small magnitude, the remaining $K-N$ higher-order DCT coefficients are safely neglected:

$$
\theta_{k} \approx \sum_{n=0}^{N-1} \psi_{k, n} x_{n}, k=0, \ldots, K-1,
$$

or, in matrix form:

$$
\boldsymbol{\theta} \approx \boldsymbol{\Psi}_{\mathrm{K}} \mathrm{X}
$$

where $(\boldsymbol{\theta})_{k}=\theta_{k},\left(\mathbf{\Psi}_{\mathbf{K}}\right)_{k, n}=\psi_{k, n}$ is the DCT basis function of order $n$ over the observation interval $(0, K-1)$ and $(\mathbf{x})_{n}=x_{n}$ is the $n$-th order DCT coefficient of $\theta_{k}$. 
The orthonormal DCT basis functions are known to perform closely to the optimal Karhunen-Loève basis functions [8] and are defined as

$$
\psi_{k, n}=\left\{\begin{array}{ll}
\sqrt{\frac{1}{K}} & n=0 \\
\sqrt{\frac{2}{K}} \cos \left(\frac{\pi n}{K}\left(k+\frac{1}{2}\right)\right) & n>0
\end{array} .\right.
$$

From the observations (1) we produce an estimate $\hat{\nu}$ of the normalized FO and an estimate $\left\{\hat{x}_{n}, n=0, \ldots, N-1\right\}$ of the DCT coefficients $\left\{x_{n}, n=0, \ldots, N-1\right\}$ using (2) with equality. The phase estimate $\hat{\theta}_{k}$ is obtained as the $K$-point inverse DCT of $\left\{\hat{x}_{n}\right\}$. Note that instead of having to estimate each of the $K$ phase samples $\phi_{k}$, the proposed method requires the estimation of only $N+1<<K$ parameters, significantly reducing the computation time.

\section{FREQUENCY OFFSET AND PHASE NOISE ESTIMATION}

The proposed iterative estimation algorithm is inspired by the SAGE algorithm, which is related to the iterative expectation maximization (EM) algorithm [9], [10]. Let us consider the case where we wish to estimate the unknown deterministic parameter vector $\mathbf{y}$ of length $M+N$ from the observations $\mathbf{r}$. The EM algorithm states that the maximum-likelihood (ML) estimate of $\mathbf{y}$ can iteratively be obtained as

$$
\hat{\mathbf{y}}^{(l)}=\arg \max _{\mathbf{y}} E_{\mathbf{a}}\left[\ln (p(\mathbf{r} \mid \mathbf{a} ; \mathbf{y})) \mid \mathbf{r} ; \hat{\mathbf{y}}^{(l-1)}\right],
$$

where $l>0$ is the iteration index and $p(\mathbf{r} \mid \mathbf{a} ; \mathbf{y})$ is the probability density function (pdf) of the received signal $r$ conditioned on the symbol sequence $\mathbf{a}$ and the parameter vector $\mathbf{y}$. The natural logarithm, $\ln (p(\mathbf{r} \mid \mathbf{a} ; \mathbf{y}))$ is called the joint $\log$-likelihood function of $\mathbf{a}$ and $\mathbf{y}$. Note that, during each iteration, the EM algorithm simultaneously computes an estimate of all components of $\mathbf{y}$. The SAGE algorithm presents an iterative framework where during each iteration, disjoint subsets of the elements of $\mathbf{y}$ are estimated separately, while keeping the corresponding complement of each subset fixed. Let us construct the vector $\mathbf{y}_{1}$ by stacking $M$ components of $\mathbf{y}$. The remaining $N$ components of $\mathbf{y}$ are used to form $\mathbf{y}_{\mathbf{2}}$. The first step of the SAGE procedure is to apply the following ML rule in order to obtain an estimate of $\mathbf{y}_{\mathbf{1}}$ :

$$
\hat{\mathbf{y}}_{\mathbf{1}}^{(l)}=\arg \max _{\mathbf{y}_{\mathbf{1}}} E_{\mathbf{a}}\left[\ln \left(p\left(\mathbf{r} \mid \mathbf{a} ; \mathbf{y}_{\mathbf{1}}, \hat{\mathbf{y}}_{\mathbf{2}}^{(l-1)}\right)\right) \mid \mathbf{r} ; \hat{\mathbf{y}}_{1}^{(l-1)}, \hat{\mathbf{y}}_{\mathbf{2}}^{(l-1)}\right],
$$

where $\hat{\mathbf{y}}_{\mathbf{2}}^{(l-1)}$ is the estimate of $\mathbf{y}_{\mathbf{2}}$ from the previous iteration. In the next step, keeping $\mathbf{y}_{\mathbf{1}}$ to the fixed value $\hat{\mathbf{y}}_{\mathbf{1}}^{(l)}$, the iterative estimate of $\mathbf{y}_{\mathbf{2}}$ is computed as:

$$
\hat{\mathbf{y}}_{\mathbf{2}}^{(l)}=\arg \max _{\mathbf{y}_{\mathbf{2}}} E_{\mathbf{a}}\left[\ln \left(p\left(\mathbf{r} \mid \mathbf{a} ; \hat{\mathbf{y}}_{\mathbf{1}}^{(l)}, \mathbf{y}_{\mathbf{2}}\right)\right) \mid \mathbf{r} ; \hat{\mathbf{y}}_{\mathbf{1}}^{(l-1)}, \hat{\mathbf{y}}_{\mathbf{2}}^{(l-1)}\right] .
$$

From (3) and (4), we note that a SAGE iteration is actually an EM iteration, where a subset of the parameter estimates is updated, while keeping the remaining parameters fixed. The estimation rules (3) and (4) are initialized by the pilotbased (PB) estimates $\hat{\mathbf{y}}_{\mathbf{1}}^{(0)}$ and $\hat{\mathbf{y}}_{\mathbf{2}}^{(0)}$. Denoting $\mathbf{r}_{\mathbf{P}}$ as the vector containing the observations (1) at the pilot symbol positions, we have:

$$
\begin{aligned}
& \hat{\mathbf{y}}_{\mathbf{1}}^{(0)}=\arg \max _{\mathbf{y}_{\mathbf{1}}} \ln \left(p\left(\mathbf{r}_{\mathbf{P}} \mid \mathbf{a}_{\mathbf{P}} ; \mathbf{y}_{\mathbf{1}}, \tilde{\mathbf{y}}_{\mathbf{2}}^{(0)}\right)\right) \\
& \hat{\mathbf{y}}_{\mathbf{2}}^{(0)}=\arg \max _{\mathbf{y}_{\mathbf{2}}} \ln \left(p\left(\mathbf{r}_{\mathbf{P}} \mid \mathbf{a}_{\mathbf{P}} ; \hat{\mathbf{y}}_{\mathbf{1}}^{(0)}, \mathbf{y}_{\mathbf{2}}\right)\right),
\end{aligned}
$$

where $\left(\mathbf{a}_{\mathbf{P}}\right)_{i}=a_{k_{i}}$ for $i=0, \ldots, K_{P}-1$ and $\tilde{\mathbf{y}}_{\mathbf{2}}^{(0)}$ is an appropriately chosen initial value of $\mathbf{y}_{\mathbf{2}}$. In the problem at hand, we wish to estimate the parameter set $\left\{\nu, x_{0}, \ldots, x_{N-1}\right\}$ from the observations (1). A straightforward partitioning would be to choose the subsets $\{\nu\}$ and $\left\{x_{0}, \ldots, x_{N-1}\right\}$, allowing us to estimate the frequency offset and the phase noise separately. Depending on the order of estimation, we choose either $\mathbf{y}_{1}=y_{1}=\nu$ and $\mathbf{y}_{\mathbf{2}}=\mathbf{x}$; or $\mathbf{y}_{\mathbf{1}}=\mathbf{x}$ and $\mathbf{y}_{\mathbf{2}}=y_{2}=\nu$.

\section{A. Phase noise estimation}

The joint log-likelihood function $\ln p(\mathbf{r} \mid \mathbf{a} ; \tilde{\nu}, \mathbf{x})$ is given by (discarding terms not depending on $\mathrm{x}$ and irrelevant multiplicative factors):

$$
\ln p(\mathbf{r} \mid \mathbf{a} ; \tilde{\nu}, \mathbf{x}) \propto \sum_{k=0}^{K-1} \Re\left[r_{k} a_{k}^{*} e^{-j\left(2 \pi \tilde{\nu} k+\theta_{k}(\mathbf{x})\right)}\right],
$$

where $\tilde{\nu}=\hat{\nu}^{(l-1)}$ if phase noise estimation precedes frequency estimation and $\tilde{\nu}=\hat{\nu}^{(l)}$ otherwise, $(\mathbf{r})_{k}=r_{k}$ and $\theta_{k}(\mathbf{x})$ indicates that the phase is a function of $\mathbf{x}$. Straightforward application of the SAGE algorithm yields the following iterative estimation rule:

$$
\hat{\mathbf{x}}^{(l)}=\arg \max _{\mathbf{x}} \sum_{k=0}^{K-1}\left|V_{k}^{(l)}\right| \cos \left(\arg \left(V_{k}^{(l)}\right)-\theta_{k}(\mathbf{x})\right),
$$

where we have defined $V_{k}^{(l)}=r_{k} e^{-j 2 \pi \tilde{\nu} k} \mu_{k}^{(l) *}$. For $k \in I_{P}$, we have $\mu_{k}^{(l)}=a_{k}$. For $k \notin I_{P}, \mu_{k}^{(l)}=E_{\mathbf{a}}\left[a_{k} \mid \mathbf{r} ; \hat{\nu}^{(l-1)}, \hat{\mathbf{x}}^{(l-1)}\right]$ is the a posteriori expectation of the symbol $a_{k}$, based on $\mathbf{r}$ and the FO estimate and DCT coefficient vector estimate from the previous iteration. Note that for $k \notin I_{P}, \mu_{k}^{(l)}$ represents the soft decision on the data symbol $a_{k}$. The non-linear character of the estimation rule (5) renders ML estimation of $\mathrm{x}$ infeasible. Assuming small additive noise, however, an approximate solution $\hat{\mathbf{x}}^{(l)}$ is obtained by minimizing $\sum_{k}\left|\arg \left(V_{k}^{(l)}\right)-\theta_{k}(\mathbf{x})\right|^{2}$ instead. With $l>0$, we obtain the following least-squares estimate:

$$
\begin{aligned}
& \hat{\mathbf{x}}^{(l)}=\left(\boldsymbol{\Psi}_{\mathbf{K}}^{\mathbf{T}} \boldsymbol{\Psi}_{\mathbf{K}}\right)^{-1} \Psi_{\mathbf{K}}^{\mathbf{T}} \mathbf{z}^{(l)}=\mathbf{\Psi}_{\mathbf{K}}^{\mathbf{T}} \mathbf{z}^{(l)} \\
& \hat{\boldsymbol{\theta}}^{(l)}=\boldsymbol{\Psi}_{\mathbf{K}} \hat{\mathbf{x}}^{(l)}=\boldsymbol{\Psi}_{\mathbf{K}} \boldsymbol{\Psi}_{\mathbf{K}}^{\mathbf{T}} \mathbf{z}^{(l)}
\end{aligned}
$$

where $\left(\mathbf{z}^{(l)}\right)_{k}=\arg \left(V_{k}^{(l)}\right)$ and the second equality in the first line of (6) follows from the orthonormality of the DCT basis functions. Similarly, the PB phase estimate is given by:

$$
\hat{\boldsymbol{\theta}}^{(0)}=\boldsymbol{\Psi}_{\mathbf{K}}\left(\boldsymbol{\Psi}_{\mathbf{P}}^{\mathbf{T}} \boldsymbol{\Psi}_{\mathbf{P}}\right)^{-\mathbf{1}} \boldsymbol{\Psi}_{\mathbf{P}}^{\mathbf{T}} \mathbf{z}^{(0)}
$$

where $\left(\mathbf{z}^{(0)}\right)_{i}=\arg \left(r_{k_{i}} e^{-j 2 \pi \tilde{\nu}^{(0)} k_{i}} a_{k_{i}}^{*}\right)$ and $\mathbf{\Psi}_{\mathbf{P}}$ is the matrix obtained by keeping only the $K_{P}$ rows of $\Psi_{\mathbf{K}}$ with pilot symbol indices. For the matrix $\Psi_{\mathrm{P}}^{\mathrm{T}} \boldsymbol{\Psi}_{\mathrm{P}}$ to be invertible, we 
need $N<K_{P}$. In [7], we propose to insert the pilot symbols at the following equidistant positions, for $i=0, \ldots, K_{P}-1$ :

$$
k_{i}=\frac{K(2 i+1)-K_{P}}{2 K_{P}},
$$

which yields a diagonal matrix $\boldsymbol{\Psi}_{\mathbf{P}}^{\mathbf{T}} \boldsymbol{\Psi}_{\mathbf{P}}=\frac{K_{P}}{K} \mathbf{I}_{\mathbf{N}}$, with $\mathbf{I}_{\mathbf{N}}$ denoting the $N \times N$ identity matrix.

Note that the argument function $\arg ($.$) in (5) returns$ values between $[-\pi, \pi]$. Consequently, when the argument of $r_{k} e^{-j 2 \pi \tilde{\nu} k} \mu_{k}^{(l) *}$ is larger (smaller) than $\pi(-\pi)$, phase wrapping occurs and the estimate is no longer accurate. We avoid this problem by unwrapping the argument function $\arg ($.$) using a phase unwrapping scheme. Although the added$ complexity is negligible, correct phase unwrapping requires the pilot symbol spacing to be sufficiently small for the PB estimate. In the remainder of this paper we assume correct phase unwrapping takes place, and $\arg ($.$) denotes the unwrapped$ argument function.

\section{B. Frequency offset estimation}

In accordance with the SAGE algorithm, the FO estimate from the $l$-th iteration is given by:

$$
\begin{aligned}
\hat{\nu}^{(l)} & =\arg \max _{\nu} \sum_{k=0}^{K-1} \Re\left[r_{k} \mu_{k}^{(l) *} e^{-j\left(2 \pi \nu k+\theta_{k}(\tilde{\mathbf{x}})\right)}\right] \\
& =\arg \max _{\nu} \sum_{k=0}^{K-1} \Re\left[u_{k}^{(l)} e^{-j 2 \pi \nu k}\right],
\end{aligned}
$$

where in the second line of (9), we have defined $u_{k}^{(l)}=$ $r_{k} \mu_{k}^{(l) *} e^{-j \theta_{k}(\tilde{\mathbf{x}})}$ with $\tilde{\mathbf{x}}=\hat{\mathbf{x}}^{(l-1)}$ when FO estimation takes place before phase noise estimation and $\tilde{\mathbf{x}}=\hat{\mathbf{x}}^{(l)}$ otherwise. In order to solve the highly non-linear equation (9), we make use of the technique proposed by Luise and Reggiannini in [5]. Defining the estimated autocorrelation function of $\left\{u_{k}^{(l)}, k=0, \ldots, K-1\right\}$ as

$$
U_{k}^{(l)}=\frac{1}{K-k} \sum_{m=k}^{K-1} u_{m}^{(l)} u_{m-k}^{(l) *},
$$

and under the assumption of high signal-to-noise ratio (SNR) and low frequency offsets, the following estimate is obtained:

$$
\hat{\nu}^{(l)}=\frac{1}{(M+1) \pi} \arg \left(\sum_{k=1}^{M} U_{k}^{(l)}\right),
$$

where $M<K$ represents the number of autocorrelation lags considered for estimation. In [5], it was shown that for large bursts $K$, choosing $M \approx K / 2$ yields close to optimum performance of the FO estimator. As the phase noise estimation algorithm requires the pilot symbols to be inserted equidistantly, the pilot-based FO estimate is given by

$$
\hat{\nu}^{(0)}=\frac{\eta}{\left(M_{P}+1\right) \pi} \arg \left(\sum_{i=1}^{M_{P}} U_{i}^{(0)}\right),
$$

where $M_{P}<K_{P}$ denotes the number of correlations that are kept for the PB FO estimate, $1 / \eta=K / K_{P}$ is the distance between consecutive pilot symbols, and $U_{i}^{(0)}$ is the estimated autocorrelation function of the sequence $\left\{r_{k} e^{-j \theta_{k}\left(\tilde{\mathbf{x}}^{(0)}\right)} a_{k}^{*}, k \in\right.$ $\left.I_{P}\right\}$. Note that the operating range of the frequency estimator is determined by the choice of $M_{P}$ and the pilot symbol ratio $\eta$. Indeed, in order that no phase wrapping takes place between consecutive pilot symbols, we need

$$
|\nu|<\frac{\eta}{\left(M_{P}+1\right)} .
$$

\section{Simulation RESUlTS}

In this section, we make use of computer simulations to assess the performance of the proposed estimation algorithm in terms of the mean-square phase error (MSPE) and the resulting bit error rate (BER) degradation ${ }^{1}$. The MSPE is defined as

$$
M S P E=\frac{1}{K} E\left[\sum_{k=0}^{K-1}\left(\phi_{k}-2 \pi \hat{\nu} k-\hat{\theta}_{k}\right)^{2}\right] .
$$

We will assume transmission of $K=100$ uncoded QPSK symbols, including $K_{P}=10$ pilot symbols arranged at positions (8), over an AWGN channel in the presence of a frequency offset and Wiener phase noise. The Wiener model is commonly used to characterize the phase noise behaviour of practical oscillators [11], [12] and is described by the following system equation:

$$
\theta_{k+1}=\theta_{k}+\Delta_{k}, k=0, \ldots, K-2 .
$$

The initial value $\theta_{0}$ is uniformly distributed in $[-\pi, \pi]$ and $\left\{\Delta_{k}\right\}$ is a sequence of i.i.d. zero-mean Gaussian random variables with variance $\sigma_{\Delta}^{2}$. Throughout this section, numerical results have been obtained for Wiener phase noise with $\sigma_{\Delta}=3^{\circ}$. Figure 1 shows the influence of the FO magnitude on the mean-square frequency estimation error $E\left[\left(\hat{\nu}^{(0)}-\nu\right)^{2}\right]$, when performing PB FO estimation using $M_{P}=2$ lags. From figure 1 , we observe that performance deteriorates significantly when $\nu$ is larger than a certain threshold value, determined by expression (13) for the operating range: $|\nu|>0.1 / 3 \approx 0.033$. We also observe that the presence of phase noise yields a degraded performance as compared to when only a static phase offset is present. In the remainder, the values $\nu$ for simulation are taken from a random distribution in $[-0.03,0.03]$ and we take $M_{P}=2$ in (12) and $M=K / 2=50$ in (11).

Two possible $\mathrm{PB}$ estimation strategies are considered. In the first approach, denoted as PBE1, the receiver computes a PB estimate of $\mathbf{x}$ using (7), with $\tilde{\nu}^{(0)}=0$ ). The resulting PB DCT coefficient estimate is then used to perform PB FO estimation (12). In the second PB estimation technique (referred to as PBE2), the estimation order is reversed: we first compute the FO estimate, taking $\tilde{\mathbf{x}}^{(0)}=\mathbf{0}$. The PB phase noise estimate from (7) is computed in the second step.

Figure 2 presents the resulting BER as a function of $E_{b} / N_{0}$, for different $N$. Also shown is the BER curve corresponding to

\footnotetext{
${ }^{1}$ The BER degradation caused by some impairment is characterized by the increase (in $\mathrm{dB}$ ) of $E_{b} / N_{0}$ (as compared to the case of no impairment) needed to maintain the BER at a specified reference level, where $E_{b}$ is the transmitted energy per bit. Throughout our discussion, we consider the BER degradation at $B E R_{\text {ref }}=10^{-4}$
} 


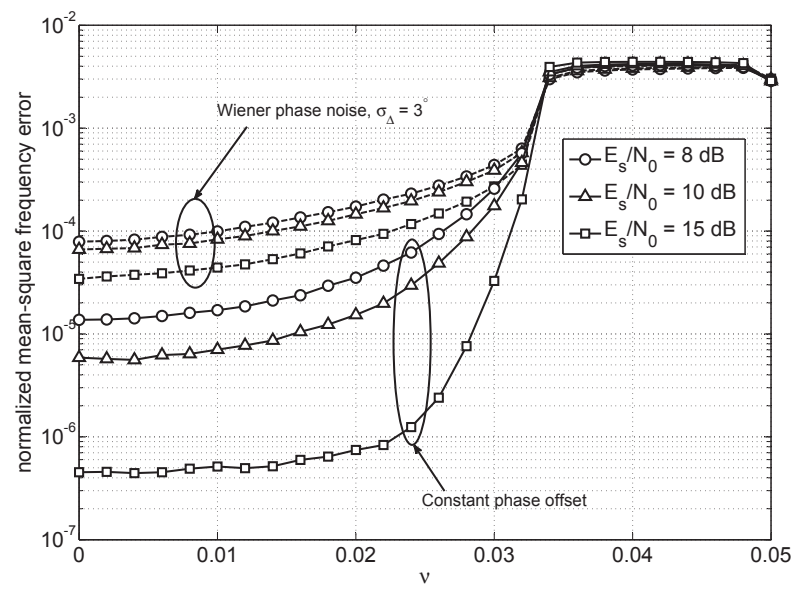

Figure 1: Performance of the PB FO estimation technique as a function of the normalized FO.

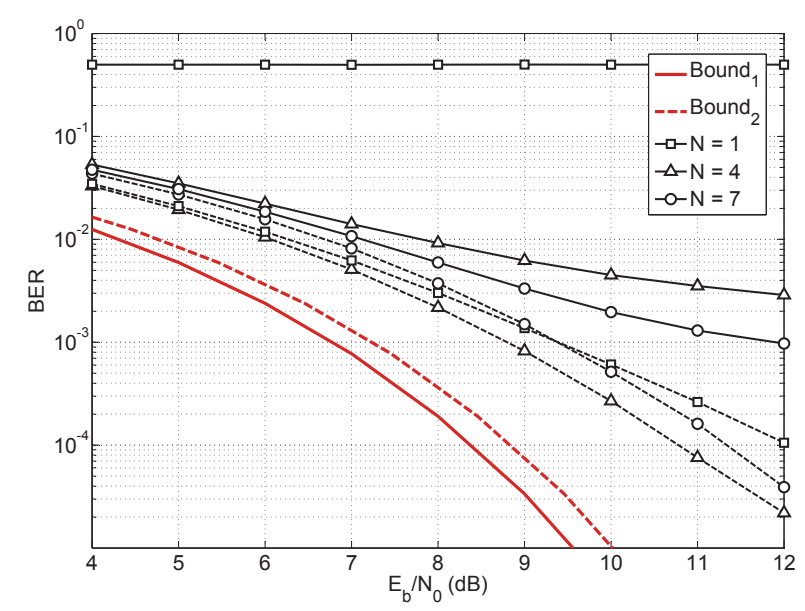

Figure 2: BER as a function of $E_{b} / N_{0}$. Solid lines: PB estimation via PBE1, dashed lines: PB estimation via PBE2.

the system with perfect synchronization when no pilot symbols are used (denoted as Bound ${ }_{1}$ in figure 2). The Bound ${ }_{2}$-curve represents the BER for perfect synchronization when the energy loss due to the pilot symbols is taken into account and shows a degradation of about $-10 \log _{10}(1-\eta)=0.46 \mathrm{~dB}$ w.r.t. Bound 1 for $\eta=0.1$. We observe that PBE2 clearly outperforms PBE1, regardless of $N$. The BER curves show a floor at high $E_{b} / N_{0}$, which is caused by neglecting the higherorder DCT coefficients in the estimation of the phase noise. However, for PBE1, the floor is much more significant and it occurs at lower values of $E_{b} / N_{0}$ as compared to PBE2. At moderate $E_{b} / N_{0}$, increasing the number of estimated DCT coefficients $N$ is beneficial for PBE1 as it yields a lower floor value, but the BER performance is still rather poor. For PBE2 however, increasing $N$ only yields improved performance up to a certain value, $N_{P B}=4$ in figure 2 . For $N>N_{P B}$, a larger amount of additive noise is included in the estimation process and the BER degradation increases.

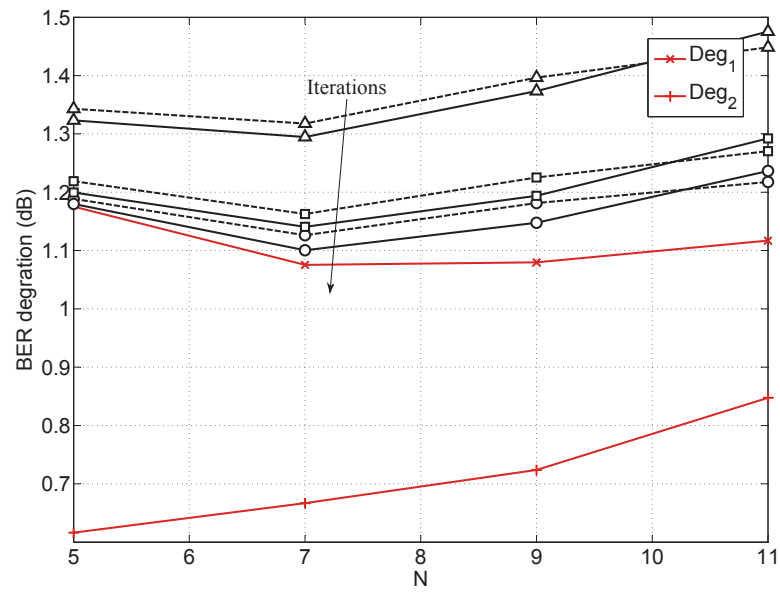

Figure 3: BER degradation as a function of $N$. Solid lines: estimation via DDE1, dashed lines: estimation via DDE2.

The degraded performance of PBE1 as compared to PBE2 can be explained as follows. When estimating the DCT coefficients of the phase noise assuming negligible FO $\left(\tilde{\nu}^{(0)}=0\right)$, $\hat{\mathbf{x}}^{(0)}$ is the PB DCT coefficient vector estimate related to the total instantaneous phase $\phi_{k}$. As $\phi_{k}$ contains a contribution that increases linearly with the time-index $k$, it does not necessarily show a low-pass behaviour. As a result, ignoring the higher-order DCT coefficients of $\phi_{k}$ results in a significant modeling error. By first estimating the FO, the linear term in $\phi_{k}$ is largely compensated for, prior to phase noise estimation, so that disregarding the higher-order DCT coefficients is justified in PBE2.

Next, we examine the BER degradation of the iterative SAGE-based estimation technique. We consider two scenarios: in the first decision-directed (DD) scheme, referred to as DDE1, the PB FO and phase noise estimates resulting from PBE2 are used to initialize the iterative FO estimation rule from (11). After 1 iteration, the resulting DD FO estimate is used to perform 1 iteration of the phase noise estimate from (6). The SAGE updating rules (11) and (6) are then iteratively repeated. It turns out that convergence is achieved after only 3 iterations. Assuming the initial PB FO estimate is sufficiently accurate, we could reduce the complexity of the estimation algorithm by skipping the updating step of the FO estimate and only refining the phase noise estimate iteratively (i.e. we only use the PB FO estimate resulting from PBE2). The corresponding estimation scheme is denoted as DDE2.

Figure 3 shows the BER degradation at a reference value $B E R_{\text {ref }}=10^{-4}$ as a function of $N$ for DDE1 and DDE2. The BER performance is compared to the case where only the phase noise is estimated when no FO is present $\left(\mathrm{Deg}_{1}\right)$. $\mathrm{Deg}_{2}$ is the BER degradation corresponding to the case where DDE2 is applied when there is no phase noise, i.e., $\sigma_{\Delta}=0^{\circ}$. From figure 3 , we observe that in the presence of phase noise, each BER degradation curve shows a minimum for an optimal value of $N\left(N_{o p t} \approx 7\right)$. Although DDE1 yields a lower BER degradation as compared to DDE2, the difference 


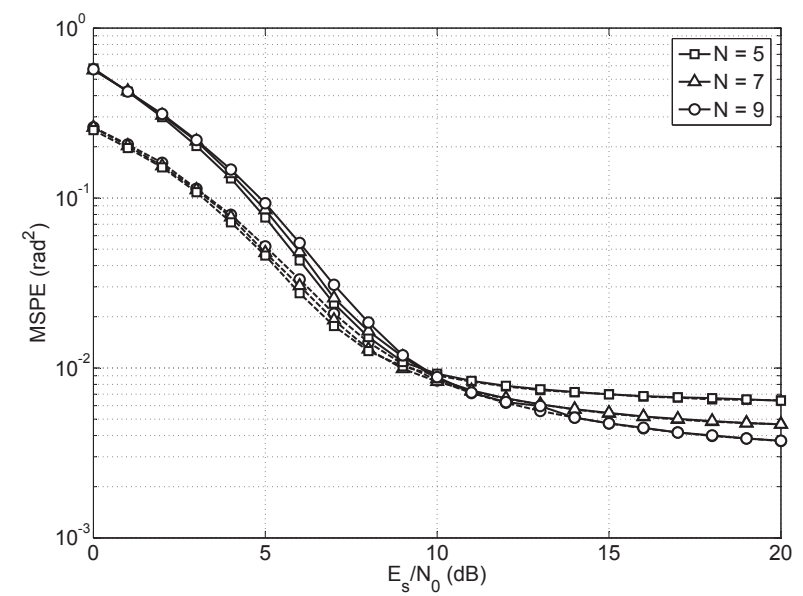

Figure 4: MSPE as a function of $E_{s} / N_{0}$. Solid lines: estimation via DDE2, dashed lines: estimation of phase noise only with perfect FO estimation.

is small. Hence, through proper selection of the number of estimated DCT coefficients, the computational complexity of the estimation procedure can be reduced by updating only the phase noise estimate, while operating at approximately the same BER. We remark here that the reduction in complexity of DDE2 as compared to DDE1 can be quite significant, since the latter method would require additional evaluation of $M$ autocorrelation samples from (10) per iteration. It is observed that the minimum BER performance is close to the $\operatorname{Deg}_{1}$ curve. Note that the added degradation of DDE1 and DDE2 as compared to $\mathrm{Deg}_{1}$ is caused by the residual FO. When no phase noise is present, it is not beneficial to estimate more than one DCT coefficient (i.e., the constant phase contribution). The BER degradation $\operatorname{Deg}_{2}$ increases as a function of $N$, since the amount of additive noise included in the phase estimate increases with $N$.

Figure 4 compares the MSPE corresponding to DDE2 as a function of $E_{s} / N_{0}$ to the MSPE when only the phase noise is estimated in the absence of a FO, for different values of $N$. The behaviour of the MSPE as a function of $N$ has been detailed extensively in our prior work [7], [13] for the case of perfect FO estimation and we observe that it is very similar when a residual FO is present. However, for low SNR, the presence of a FO leads to a degraded MSPE performance, since the FO estimate is less accurate in the low $E_{s} / N_{0}$ region. For high $E_{s} / N_{0}$, DDE2 yields the same MSPE performance as when no FO is present. Simulation results not shown here, indicate that the MSPE resulting from DDE1 coincides with the MSPE resulting from DDE2, implying that the DCT-based phase noise estimation algorithm is quite robust against the residual FO remaining after PB FO estimation.

\section{CONCLUSIONS}

We have discussed a low-complexity iterative decisiondirected FO and phase noise estimation technique that is based on the SAGE algorithm. The phase noise estimate is obtained through a truncated DCT model of the phase, where the higherorder DCT coefficients are neglected. An estimate of the FO is computed, based on the estimator presented in [5], which is derived from the ML criterion under the assumption of high signal-to-noise ratio. The performance of the proposed scheme is evaluated by means of computer simulations. The following pilot-based (PB) scheme is adopted for initialization: assuming no phase noise is present the PB FO estimate is computed. The resulting $\mathrm{PB}$ FO estimate is used to compute the $\mathrm{PB}$ phase noise estimate. Both the FO and phase noise estimates are iteratively refined in accordance with the SAGE framework. Numerical results show that instead of also computing the iterative FO estimate, comparable performance can be achieved by only updating the phase noise estimate iteratively using the PB FO estimate, which yields reduced complexity. For the considered simulation set-up, convergence of the SAGE algorithm is achieved after only 3 iterations.

\section{ACKNOWLEDGEMENT}

The authors wish to acknowledge the activity of the Network of Excellence in Wireless COMmunications NEWCOM++ of the European Commission (contract no. 216715) that motivated this work. The second author would like to acknowledge the financial support of the Fund for Scientific Research - Flanders (FWO Vlaanderen).

\section{REFERENCES}

[1] T.H. Lee and A. Hajimiri, "Oscillator phase noise: A tutorial," IEEE Journal of Solid-State Circuits, vol. 35, no. 3, pp. 326-336, March 2000

[2] S. Kay, "A fast and accurate single frequency estimator," IEEE Trans. Acoust., Speech, Sign. Proc., vol. 37, pp. 1987 - 1990, December 1989.

[3] M.P. Fitz, "Further results in the fast estimation of a single frequency," IEEE Trans. Comm., vol. 42, no. 2/3/4, pp. 862 - 864, February/March/April 1994.

[4] H. Meyr, M. Moeneclaey, and S. Fechtel, Digital Communication Receivers : Synchronization, Channel Estimation and Signal Processing, Wiley Series in Telecommunications and Signal Processing, USA, 1998.

[5] M. Luise and R. Reggiannini, "Carrier frequency recovery in all-digital modems for burst-mode transmissions," IEEE Trans. Comm., vol. 43, no. 2/3/4, pp. 1169 - 1178, February/ March/ April 1995.

[6] J. Fessler and A. Hero, "Space-alternating generalized expectationmaximization algorithm," IEEE Trans. Sign. Proc., vol. 42, no. 10, pp. 2664 - 2677, October 1994.

[7] J. Bhatti and M. Moeneclaey, "Iterative soft-decision-directed phase noise estimation from a DCT basis expansion," in Proceedings of PIMRC 2009, Tokyo, Japan, September 2009.

[8] T. Ahmed, N. Natarajan and K.R. Rao, "Discrete cosine transform," IEEE Trans. Computers, vol. 23, no. 1, pp. 90 - 93, January 1974.

[9] A.P. Dempster, N.M. Laird, and D.B. Rubin, "Maximum likelihood from incomplete data via the EM algorithm," Journal of the Royal Statistical Society. Series B (Methodological), vol. 39, no. 1, pp. 1-38, 1977.

[10] R. Boyles, "On the convergence of the EM algorithm," Journal of the Royal Statistical Society. Series B, , no. 1, pp. 47-50, 1983.

[11] G. Colavolpe, A. Barbieri, and G. Caire, "Algorithms for iterative decoding in the presence of strong phase noise," IEEE Journal on Selected Areas in Communications, vol. 23, pp. 1748-1757, September 2005.

[12] S. Bay, C. Herzet, J. Brossier, J. Barbot, and B. Geller, "Analytical and asymptotic analysis of Bayesian Cramer-Rao bound for dynamical phase offset estimation," IEEE Transactions on Signal Processing, vol. 56, no. 1, pp. 61-70, January 2008.

[13] J. Bhatti and M. Moeneclaey, "Performance analysis of iterative decision-directed phase noise estimation," in Future Network and Mobile Summit 2010 Conference Proceedings, Florence, Italy, June 2010. 